\title{
Mediterranean diet adherence during pregnancy and risk of wheeze and eczema in the first year of life: INMA (Spain) and RHEA (Greece) mother-child cohort studies
}

Leda Chatzi $^{1 *}$, Raquel Garcia ${ }^{2,3}$, Theano Roumeliotaki ${ }^{1}$, Mikel Basterrechea ${ }^{3,4,5}$, Haizea Begiristain ${ }^{5}$, Carmen Iñiguez ${ }^{3,6,7}$, Jesus Vioque ${ }^{3,8}$, Manolis Kogevinas ${ }^{2,3,9,10}$ and Jordi Sunyer ${ }^{2,3,10,11}$ on behalf of the INMA and RHEA study groups

${ }^{1}$ Department of Social Medicine, Faculty of Medicine, University of Crete, PO Box 2208, Heraklion, 71003 Crete, Greece

${ }^{2}$ Centre for Research in Environmental Epidemiology (CREAL), Barcelona, Spain

${ }^{3}$ CIBER Epidemiologia y Salud Pública (CIBERESP), Barcelona, Spain

${ }^{4}$ Public Health Division of Gipuzkoa, Basque Government, San Sebastian, Spain

${ }^{5}$ Instituto Investigation BioDonostia, Basque Government, San Sebastian, Spain

${ }^{6}$ Public Health Research Institute, Valencia, Spain

${ }^{7}$ Universitat de Valenica, Valencia, Spain

${ }^{8}$ Departamento de Salud Publica, Universidad Miguel Hernandez, Alicante, Spain

${ }^{9}$ National School of Public Health, Athens, Greece

${ }^{10}$ Municipal Institute of Medical Research, Barcelona, Spain

${ }^{11}$ Universitat Pompeu Fabra, Barcelona, Spain

(Submitted 7 September 2012 - Final revision received 19 March 2013 - Accepted 26 March 2013 - First published online 17 May 2013 )

\begin{abstract}
Maternal diet during pregnancy might influence the development of childhood allergic disorders. The aim of the present study was to evaluate the impact of Mediterranean diet (MD) adherence during pregnancy on wheeze and eczema in the first year of life in two population-based mother-child cohorts in Spain and Greece. We studied 1771 mother-newborn pairs from the Spanish multi-centre 'INMA' (INfancia y Medio Ambiente) study (Gipuzkoa, Sabadell and Valencia) and 745 pairs from the 'RHEA' study in Crete, Greece. The symptoms of wheeze and eczema were based on the criteria of the International Study of Asthma and Allergies in Childhood. Maternal diet during pregnancy was assessed by FFQ and MD adherence was evaluated through an a priori score. Multivariate log-binomial regression models were used to adjust for several confounders in each cohort and summary estimates were obtained by a meta-analysis. MD adherence was not associated with the risk of wheeze and eczema in any cohort, and similar results were identified in the meta-analysis approach. High meat intake (relative risk (RR) 1.22, $95 \%$ CI 1.00, 1.49) and 'processed' meat intake (RR 1.18, 95\% CI 1.02, 1.37) during pregnancy were associated with an increased risk of wheeze in the first year of life, while a high intake of dairy products was significantly associated with a decreased risk of infantile wheeze (RR $0.83,95 \%$ CI $0.72,0 \cdot 96$ ). The results of the present study show that high meat intake during pregnancy may increase the risk of wheeze in the first year of life, while a high intake of dairy products may decrease it.
\end{abstract}

Key words: Mediterranean diet: Pregnancy: Wheeze: Eczema: Infants

Prenatal life is a critical period for the development of the immune system, and maternal diet during pregnancy has been proposed to influence fetal immune responses that might predispose to childhood allergic manifestations ${ }^{(1-3)}$. Prospective cohort studies of diet and respiratory and atopic diseases have focused largely on relationships with intakes of individual nutrients and foods or food groups, with several aspects of maternal diet being hypothesised to influence the development of wheeze and atopic diseases in children aged 2-8 years, including the intake of fruit ${ }^{(4,5)}$, fish ${ }^{(6-8)}$, $n-3$ fatty acids ${ }^{(9)}$, and some vitamins and antioxidants with conflicting results ${ }^{(10-14)}$. However, these studies may have failed to account for the interactions between nutrients and have not taken into consideration that some nutrients are

Abbreviations: EU, European Union; INMA, INfancia y Medio Ambiente; ISAAC, International Study of Asthma and Allergies in Childhood; MD, Mediterranean diet; RR, Relative risk. 
intercorrelated $^{(15)}$. Thus, interest has shifted to place greater emphasis on dietary patterns that represent a broader picture of food and nutrient consumption and may therefore be more predictive of disease risk.

There are few studies that have evaluated dietary patterns in pregnant populations in association with respiratory and atopic outcomes in early childhood with conflicting results. The Avon Longitudinal Study of Parents and Children (ALSPAC) in the UK and the longitudinal pregnancy cohort study Project Viva in the USA have shown no relationships between dietary patterns in pregnancy and asthma-related symptoms at 7 and 3 years of age, respectively ${ }^{(16,17)}$. A birth cohort study carried out in Menorca (part of the Spanish INfancia y Medio Ambiente (Environment and Childhood) (INMA) birth cohort study) has shown that higher maternal adherence to a Mediterranean diet (MD) during pregnancy is significantly related to a reduced risk of wheeze and atopy in children aged 6.5 years $^{(18)}$. On the contrary, data from a birth cohort study carried out in Japan have shown that maternal Western diet, characterised by a high intake of vegetable oil, salt-containing seasonings, red meat, eggs, chicken and white vegetables, was significantly associated with a reduced risk of wheeze in children aged 16-24 months ${ }^{(19)}$. To date, there are no studies on the effect of dietary patterns in pregnancy on respiratory and atopic outcomes in the first year of life.

The objective of the present study was to determine the association between adherence to a MD in pregnancy, as measured by a widely used dietary score, and the risk of wheeze and eczema in the first year of life in two large population-based mother-child cohorts in the Mediterranean area: the INMA study, which is a collaborative mother-child cohort study that was established in Spain in $2003^{(20)}$, and the RHEA study in Crete, Greece, a population-based mother-child cohort study initiated in Crete in $2007^{(21)}$.

\section{Methods}

\section{Study population}

INfancia y Medio Ambiente Cohort. The INMA Project is a multi-centre population-based mother and child cohort study established in several geographical areas in Spain ${ }^{(20)}$. At their first routine specialised antenatal care visit to the main public hospital or health centre of reference, 2150 pregnant women were recruited from three study areas (855 from Valencia, 657 from Sabadell and 638 from Gipuzkoa). Recruitment was done from November 2003 to June 2005 in Valencia, from July 2004 to July 2006 in Sabadell and from April 2006 to January 2008 in Gipuzkoa. In Spain, 70-90\% of women use the universal public health care services during pregnancy ${ }^{(22)}$. Overall, $60 \%$ of pregnant women who were selected agreed to participate in the study. Women who experienced spontaneous or induced abortions ( $n$ 67) or gave birth to stillborn infants ( $n$ 18) were excluded from the study. Questionnaires regarding health behaviours, sociodemographic characteristics, pregnancy history and other factors were administered by trained interviewers at recruitment, during the third trimester and at delivery. In the present analysis, we included only those women who had completed a FFQ in the first trimester and their infants had available data on respiratory outcomes ( $n$ 1784, 83\%). Due to implausible values for total energy intake (outside the range of $4184-16736 \mathrm{~kJ} / \mathrm{d}$ ), thirteen additional women were excluded from the analysis ${ }^{(23)}$. Thus, the final analysis was based on 1771 children (694 in Valencia, 535 in Gipuzkoa and 542 in Sabadell). The study was conducted according to the guidelines laid down in the Declaration of Helsinki and all procedures involving human subjects were approved by the ethical committee of the Institut Municipal d'Investigació Mèdica of Barcelona and by the ethical committees of the hospitals involved in the study. Written informed consent was obtained from all women participating in the cohort study.

RHEA cohort. The 'RHEA' project is a mother-child study that examines prospectively a population-based cohort of pregnant women and their children in the prefecture of Heraklion, Crete ${ }^{(24)}$. Female residents (Greek and immigrants) who became pregnant during the 12-month period from February 2007 until February 2008 were contacted and asked to participate in the study. The first contact was made at the time of the first major ultrasound examination, about week 15 of gestation or earlier. Face-to-face structured questionnaires together with self-administered questionnaires and medical records were used to obtain information on several dietary, environmental and psychosocial exposures during pregnancy and early childhood.

During the study recruitment period, 1765 eligible women were approached, 1610 (91\%) agreed to participate and 1317 (82\%) were followed through delivery. Only singleton pregnancies were included in this analysis ( $n$ 1281). Women who experienced spontaneous or induced abortions ( $n$ 51) or gave birth to stillborn infants ( $n$ 2) were excluded, as were those women with incomplete information on reproductive outcomes ( $n$ 32) for any of the following reasons: change of address or delivery outside Heraklion prefecture and/or incomplete or missing medical records. A total of 798 (67\%) participants provided complete questionnaire data on diet during pregnancy and health outcomes. Due to implausible values for total energy intake (outside the range of $4184-16736 \mathrm{~kJ} / \mathrm{d}$ ), fifty-three women were excluded from the analysis ${ }^{(23)}$. Hence, a cohort of 745 women was available for this analysis. The study was conducted according to the guidelines laid down in the Declaration of Helsinki and all procedures involving human subjects were approved by the ethical committee of the University Hospital in Heraklion, Crete, Greece. Written informed consent was obtained from all women participating in the study.

\section{Dietary intake during pregnancy}

INfancia y Medio Ambiente cohort. In the INMA cohort, a FFQ was administered by trained interviewers, with 100 food items to assess usual food and nutrient intakes during the first trimester of pregnancy. The questionnaire asked how often, on average, the participants had consumed a particular amount of a specific type of food from the last 
menstrual period until the time of the interview (mean 13.8 (SD $2.5)$ weeks of gestation). The FFQ was an adapted version of Willett's questionnaire ${ }^{(25)}$ that was developed and validated in a group of pregnant women of the INMA study in Valencia ${ }^{(26)}$. Standard units or reference serving sizes were specified for each food item. The questionnaire had nine possible intake frequency categories, ranging from 'never or less than once per month' to ' 6 or more times per day'. The intake frequency for each food item was converted to the average daily intake for each participant.

RHEA cohort. The RHEA FFQ was administered in mid-pregnancy (14th-18th weeks of gestation, mean 14.6 (SD 3.2)) assessing dietary habits over pregnancy ${ }^{(27)}$. This is a semi-quantitative questionnaire, containing 250 food items with the following components: intake frequency; dietary supplements; information on organic food consumption; different types of vegetarianism; dietary changes due to pregnancy; conditions of pregnancy that could affect diet and food intake (nausea, vomiting, etc.). The questions were hierarchical, from general to more specific. For each food item, the participants were asked about both frequency of consumption and average portion size. The exact frequency of consumption was given per day, per week and/or per month, depending on the food item. Photographs were used to visualise small, medium and large portion sizes for each food item and the respondents had to choose one of the three pictures. The FFQ used in the RHEA cohort was validated with a series of three $24 \mathrm{~h}$ recalls in a subsample of ninety-seven women at 14-18 weeks of gestation ${ }^{(27)}$.

The intake frequency for each food item was converted to the average daily intake for each participant. For complex items (such as mixed dishes), standard recipes were used when available, otherwise new recipes that matched the description of the food were made and the intake of foods from different items was aggregated.

Mediterranean diet during pregnancy. To evaluate adherence to a MD during pregnancy, we used a score applied in a large cohort study (European Prospective Investigation into Cancer and Nutrition; EPIC) in adults ${ }^{(28)}$. For beneficial components (vegetables, legumes, fruits and nuts, cereals, fish and seafood, and dairy products), women whose consumption was below the median (cohort-specific median) were assigned a value of 0 and women whose consumption was at or above the median were assigned a value of 1 . For components presumed to be detrimental (meat, including all types of meat), women whose consumption was below the median were assigned a value of 1 , whereas women whose consumption was at or above the median were assigned a value of $0^{(28)}$. For fat intake (the eighth food category), we used the ratio of daily consumption of monounsaturated lipids to that of saturated lipids ${ }^{(28)}$. Because the score had been developed for adults and the present study population involved pregnant women, we presumed dairy products to be protective and not detrimental dietary compounds and did not include alcohol consumption in the score ${ }^{(29)}$. The total MD score ranged from 0 (minimal adherence to the traditional MD) to 8 (maximal adherence). The score was categorised to reflect three levels of adherence: (1) $\leq 3$, low; (2) 4-5, medium; (3) 6-8, high MD quality in each cohort separately.

\section{Wheeze and eczema in infancy}

INfancia y Medio Ambiente cohort. Information about wheeze and eczema in the first year of life was obtained from parents through interviewer-administered questionnaires at the first year of life (mean 13.6 (SD 1.4) months) based on the International Study of Asthma and Allergies in Childhood (ISAAC) phase-I questionnaire ${ }^{(30)}$. Wheezing was defined as 'whistling or wheezing from the chest, but not noisy breathing from the nose since birth'. Occurrence of eczema was defined as a positive answer to the question 'Has a doctor ever told you that your son/daughter has had atopic eczema?'.

RHEA cohort. Parents were contacted 9 months after birth (mean 9.4 (SD 1.9) months) for a structured telephonic interview to record information on the symptoms of wheeze and eczema based on the ISAAC phase-I questionnaire ${ }^{(30)}$. Wheeze was defined as present if the parent answered 'yes' to the question 'Has your child ever had wheezing or whistling in the chest since birth?'. Eczema was defined as a positive answer to the question 'Has a doctor ever told you that your child has had atopic dermatitis/eczema?'.

\section{Potential confounders}

Potential confounders included characteristics that have an established or potential association with wheeze, eczema in the first few months of life and MD in pregnancy including the following: maternal and paternal age; maternal and paternal education (low level: $\leq 6$ years of school, medium level: $>6$ and $\leq 12$ years of school and high level: university of technical college degree); maternal pre-pregnancy weight $(\mathrm{kg})$; maternal pre-pregnancy and paternal BMI $\left(\mathrm{kg} / \mathrm{m}^{2}\right)$; weight gain during pregnancy $(\mathrm{kg})$; maternal smoking during pregnancy (yes/no); physical activity during pregnancy (yes/no); total energy intake during pregnancy $(\mathrm{kJ} / \mathrm{d}$ for the first trimester in the INMA cohort and for mid-pregnancy for the RHEA cohort); parity (primiparous or multiparous); infant sex (male or female); maternal and paternal history of asthma (yes/no); maternal and paternal history of eczema (yes/ no); birth weight $(\mathrm{g})$; preterm birth (yes/no); breast-feeding duration (months); damp stains or mould spots in the child's bedroom (yes/no); pet ownership since birth (yes/no); daycare attendance in the first year of life (yes/no); exposure to passive smoking in the first year of life (yes/no).

\section{Statistical analysis}

Data analysis was performed using STATA version 10.0 (StataCorp LP). The primary outcome variables of interest were wheeze and eczema in the first 1 year of life. The primary exposure of interest was the adherence to a MD during pregnancy. Differences in the baseline characteristics of the study participants between cohorts and bivariate associations between dependent and independent variables were studied using Pearson's $\chi^{2}$ test for categorical variables or 
the Kruskal-Wallis test for continuous ones. The comparisons between mean MD scores across cohorts were performed using a one-way ANOVA with Scheffe's correction for multiple comparisons.

Multivariable log-binomial regression models were further used to examine the association between a MD in pregnancy and the outcomes of interest after adjusting for confounders. Variables that were associated $(P<0 \cdot 1)$ with both the outcome and exposure or that were considered $a$ priori to be important confounders were included in the multivariate model: maternal age; education; maternal history of asthma; smoking during pregnancy; parity; duration of breast-feeding; child's age at assessment; child's sex. For eczema, maternal history of eczema was used instead of maternal history of asthma in the multivariate models.

Cohort-specific relative risks (RR) with $95 \%$ CI were computed to estimate the degree of association. The heterogeneity of the effects across the four study areas was assessed by a meta-analysis. Random-effects pooled estimates and $P$ value of the $\chi^{2}$ test for heterogeneity are reported. Statistically significant heterogeneity was considered when the $P$ value was below $0 \cdot 10$. Association testing was conducted assuming a 0.05 significance level and a two-sided alternative hypothesis.

\section{Results}

The distribution of baseline characteristics of mother-child pairs participating in the Spanish INMA cohort and the Cretan cohort (RHEA) studies is given in Table 1. Women in the RHEA cohort were younger $(P<0 \cdot 001)$, heavier $(P<0 \cdot 001)$, more likely to be multiparous $(P<0 \cdot 001)$ and more exposed to smoke during pregnancy compared with the Spanish cohorts $(P<0 \cdot 001)$. Children from the INMA-Gipuzkoa cohort (Atlantic area of Spain) had higher

Table 1. Baseline characteristics of the study participants in the INMA (INfancia y Medio Ambiente) and RHEA mother-child cohort studies (Mean values with their standard errors; number of participants and percentages)

\begin{tabular}{|c|c|c|c|c|c|c|c|c|c|}
\hline & \multicolumn{2}{|c|}{$\begin{array}{l}\text { INMA-Gipuzkoa } \\
\quad(n \text { 535) }\end{array}$} & \multicolumn{2}{|c|}{$\begin{array}{l}\text { INMA-Saba- } \\
\text { dell }(n 542)\end{array}$} & \multicolumn{2}{|c|}{$\begin{array}{l}\text { INMA-Valencia } \\
\quad(n \text { 694) }\end{array}$} & \multicolumn{2}{|c|}{$\begin{array}{l}\text { RHEA } \\
(n 745)\end{array}$} & \multirow[b]{2}{*}{$P^{*}$} \\
\hline & $n$ & $\%$ & $n$ & $\%$ & $n$ & $\%$ & $n$ & $\%$ & \\
\hline \multicolumn{10}{|l|}{ Mother and family characteristics } \\
\hline Maternal age at enrolment (years) & \multirow{2}{*}{\multicolumn{2}{|c|}{$32 \cdot 6$}} & \multirow{2}{*}{\multicolumn{2}{|c|}{31.6}} & & & & & $<0.001$ \\
\hline Mean & & & & & \multicolumn{2}{|c|}{$31 \cdot 3$} & \multicolumn{2}{|c|}{$29 \cdot 7$} & \\
\hline SE & \multicolumn{2}{|c|}{0.2} & \multicolumn{2}{|c|}{0.2} & \multicolumn{2}{|c|}{0.2} & \multicolumn{2}{|c|}{0.2} & \\
\hline Pre-pregnancy BMI (kg/m²) & & \multirow{2}{*}{\multicolumn{2}{|c|}{$23 \cdot 8$}} & & & & & $<0.001$ \\
\hline Mean & \multirow{2}{*}{\multicolumn{2}{|c|}{$\begin{array}{r}23.0 \\
0.2\end{array}$}} & & & \multicolumn{2}{|c|}{$23 \cdot 8$} & \multicolumn{2}{|c|}{$24 \cdot 3$} & \\
\hline SE & & & \multicolumn{2}{|c|}{0.2} & \multicolumn{2}{|c|}{0.2} & \multicolumn{2}{|c|}{0.2} & \\
\hline Maternal education & & & & & & & & & $<0.001$ \\
\hline Low & 73 & $13 \cdot 7$ & 140 & $25 \cdot 9$ & 217 & 31.3 & 133 & $18 \cdot 0$ & \\
\hline Medium & 192 & $36 \cdot 0$ & 233 & $43 \cdot 1$ & 301 & 43.4 & 372 & $50 \cdot 3$ & \\
\hline High & 269 & $50 \cdot 4$ & 167 & $30 \cdot 9$ & 176 & $25 \cdot 4$ & 235 & $31 \cdot 8$ & \\
\hline Maternal smoking during pregnancy & 127 & 24.4 & 152 & 28.6 & 274 & 39.5 & 268 & $36 \cdot 3$ & $<0.001$ \\
\hline Exposure to passive smoke at home during pregnancy & 77 & $14 \cdot 8$ & 183 & 34.5 & 317 & $45 \cdot 9$ & 576 & $78 \cdot 0$ & $<0.001$ \\
\hline Parity, multiparous & 213 & 41.8 & 214 & 41.4 & 305 & 44.5 & 430 & $57 \cdot 7$ & $<0.001$ \\
\hline Maternal history of eczema & 32 & $6 \cdot 0$ & 71 & $13 \cdot 1$ & 65 & $9 \cdot 4$ & 122 & $16 \cdot 9$ & $<0.001$ \\
\hline Maternal history of asthma & 31 & $5 \cdot 8$ & 45 & $8 \cdot 3$ & 50 & $7 \cdot 2$ & 23 & $3 \cdot 3$ & $<0.001$ \\
\hline Paternal history of eczema & 18 & 3.4 & 39 & $7 \cdot 2$ & 33 & 4.8 & 48 & $6 \cdot 8$ & 0.015 \\
\hline Paternal history of asthma & 38 & $7 \cdot 1$ & 36 & 6.6 & 36 & $5 \cdot 2$ & 21 & $2 \cdot 9$ & 0.004 \\
\hline \multicolumn{10}{|l|}{ Child characteristics } \\
\hline Sex (male) & 269 & $50 \cdot 7$ & 282 & $52 \cdot 2$ & 367 & $52 \cdot 9$ & 388 & $52 \cdot 1$ & 0.894 \\
\hline Gestational age at birth (weeks) & & & & & & & & & $<0.001$ \\
\hline Mean & \multicolumn{2}{|c|}{$39 \cdot 8$} & & & & & & & \\
\hline SE & & & & & & & & 1 & \\
\hline Birth weight $(\mathrm{g})$ & & & & & & & & & 0.004 \\
\hline Mean & & & & & & & & & \\
\hline SE & & & & & & 8 & & 6.9 & \\
\hline Breast-feeding duration (months) & & & & & & & & & $<0.001$ \\
\hline Mean & & & & & & & & & \\
\hline SE & & & & & & & & & \\
\hline Exposure to passive smoke in the first year of life & 206 & $40 \cdot 8$ & 264 & $51 \cdot 2$ & 397 & 58.4 & 413 & $56 \cdot 0$ & $<0.001$ \\
\hline Daycare attendance in the first year of life & 239 & $47 \cdot 2$ & 161 & $31 \cdot 3$ & 141 & $20 \cdot 7$ & 23 & $3 \cdot 1$ & $<0.001$ \\
\hline Dampness in the house in the first year of life & 33 & $6 \cdot 2$ & 23 & 4.3 & 52 & 7.5 & 187 & $25 \cdot 8$ & $<0.001$ \\
\hline Pet ownership in the first year of life & 84 & $15 \cdot 9$ & 178 & $33 \cdot 6$ & 323 & $46 \cdot 9$ & 90 & $12 \cdot 1$ & $<0.001$ \\
\hline Outcomes in the first year of life & & & & & & & & & \\
\hline Age at assessment (months) & & & & & & & & & $<0.001$ \\
\hline Mean & & & & & & & & & \\
\hline SE & & & & & & & & & \\
\hline Wheeze ever & 192 & $35 \cdot 9$ & 206 & $38 \cdot 6$ & 175 & $25 \cdot 2$ & 195 & $26 \cdot 2$ & $<0.001$ \\
\hline Eczema & 86 & $16 \cdot 1$ & 123 & 23.5 & 113 & $16 \cdot 4$ & 104 & $14 \cdot 1$ & $<0.001$ \\
\hline
\end{tabular}

*Kruskal-Wallis test for continuous covariates; $\chi^{2}$ test for categorical variables. 
gestational age $(P<0.001)$ and birth weight $(P=0.004)$, higher rates of breast-feeding $(P<0.001)$, daycare attendance $(P<0.001)$ and less exposure to passive smoking $(P<0.001)$ in the first year of life compared with children from the Mediterranean areas of Spain (Valencia and Sabadell) and the RHEA cohort in Crete.

The mean age of assessment of respiratory and atopic outcomes in the first year was higher in the INMA-Gipuzkoa (14.3 (SD 0.05) months) and INMA-Sabadell (14.5 (SD 0.03) months) cohorts than in the INMA-Valencia (12.5 (SD 0.04) months) and RHEA (9.4 (SD 0.07) months) cohorts (Table 1). Accordingly, the prevalence of wheeze was higher in the INMA-Gipuzkoa (35.9\%) and INMA-Sabadell (38.6\%) cohorts than in the INMA-Valencia (25.2\%) and RHEA (26.2\%) cohorts $(P<0 \cdot 001)$. The prevalence of eczema was higher in the INMA-Sabadell cohort (23.5\%) than in the other cohorts $(P<0 \cdot 001)$.

Food group intakes during pregnancy differed significantly across cohorts, except vegetable intake (Fig. 1). A higher intake of fruits and nuts during pregnancy was reported in the RHEA cohort (median 408.5 g; $P<0.05$ ), a higher intake of meat was reported in the Valencia $(128.3 \mathrm{~g})$ and Sabadell $(123.2 \mathrm{~g})$ cohorts $(P<0.05)$, and the lowest fish intake (median 19.8g) and legume intake (median $7.9 \mathrm{~g}$ ) were reported in the RHEA cohort $(P<0.05)$. Dairy product intake was higher in the INMA-Gipuzkoa and INMA-Valencia cohorts than in the INMA-Sabadell and RHEA cohorts $(P<0.05)$, while a higher intake of MUFA was reported in the INMA-Sabadell and INMA-Valencia cohorts than in the other two cohorts $(P<0 \cdot 05)$.

The distribution of MD scores differed significantly between each pair of cohorts $(P<0 \cdot 05)$, while only the INMA-Sabadell and INMA-Valencia cohorts had similar distributions (Fig. 2). The highest proportion of subjects with a low adherence to the MD (65.4\%) was observed in the INMA-Gipuzkoa cohort (mean MD score 3.0 (SD 1.3)), while the highest proportion of subjects with a high adherence to the MD was observed in the RHEA cohort (11.5\%, mean MD score 3.5 (SD 1.6)).

Table 2 presents the associations between wheeze and eczema in the first year of life and MD score during pregnancy in the INMA and RHEA cohorts after adjusting for several confounders. A high adherence to the MD was not significantly associated with wheeze or eczema in the first year of life in any cohort, and similar results were identified in the meta-analysis approach (wheeze: RR 0.97, 95\% CI 0.77, 1·24, $P$ for heterogeneity $=0.437$; eczema: RR $1 \cdot 22,95 \%$ CI $0 \cdot 87$, $1.70, P$ for heterogeneity $=0.581$; Fig. 3). Further analysis by food group intake revealed that high (third tertile $v$. first tertile) meat intake (RR 1.22, 95\% CI 1.00, 1.49, $P$ for heterogeneity $=0 \cdot 167)$ and processed meat intake (RR 1.18, $95 \%$ CI $1 \cdot 02,1 \cdot 37, P$ for heterogeneity $=0.650)$ during pregnancy were associated with an increased risk of wheeze in the first year of life. There was statistically significant heterogeneity of results on high (third $v$. first tertile) fish intake between the study areas ( $P$ for heterogeneity=0.036): in the INMA-Valencia (RR 1.28, 95\% CI 0.94, 1.73) and RHEA (RR 1.41, 95\% CI 1.04, 1.90) cohorts, the associations with wheeze in the first year of life were positive, whereas in the INMA-Gipuzkoa (RR 0.85, 95\% CI 0.64, 1.13) and INMASabadell (RR 0.90, 95\% CI 0.69, 1.18) cohorts, they were negative, though not statistically significant. A high intake of dairy products (milk, cheese and yogurt) during pregnancy was associated with a decreased risk of wheeze in the first year of life in the different cohorts and in the pooled

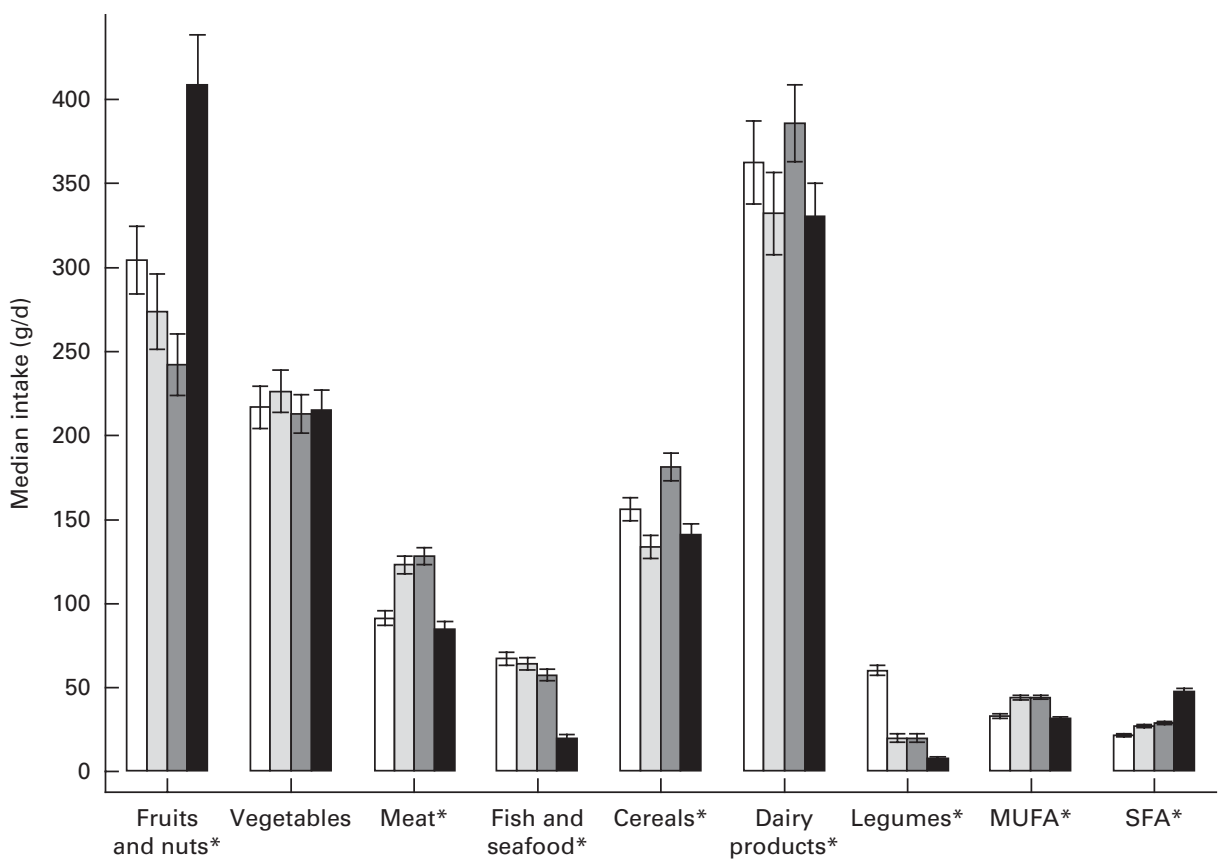

Fig. 1. Food groups intake during pregnancy in the INMA (INfancia y Medio Ambiente) and RHEA mother-child cohort studies. Values are medians, with their $95 \% \mathrm{Cl}$ represented by vertical bars. Values were significantly different $(P<0.05$; non-parametric equality-of-medians test). $\square$, INMA-Gipuzkoa; $\square$, INMA-Sabadell; $\square$, INMA-Valencia; $\mathbf{0}$, RHEA. 


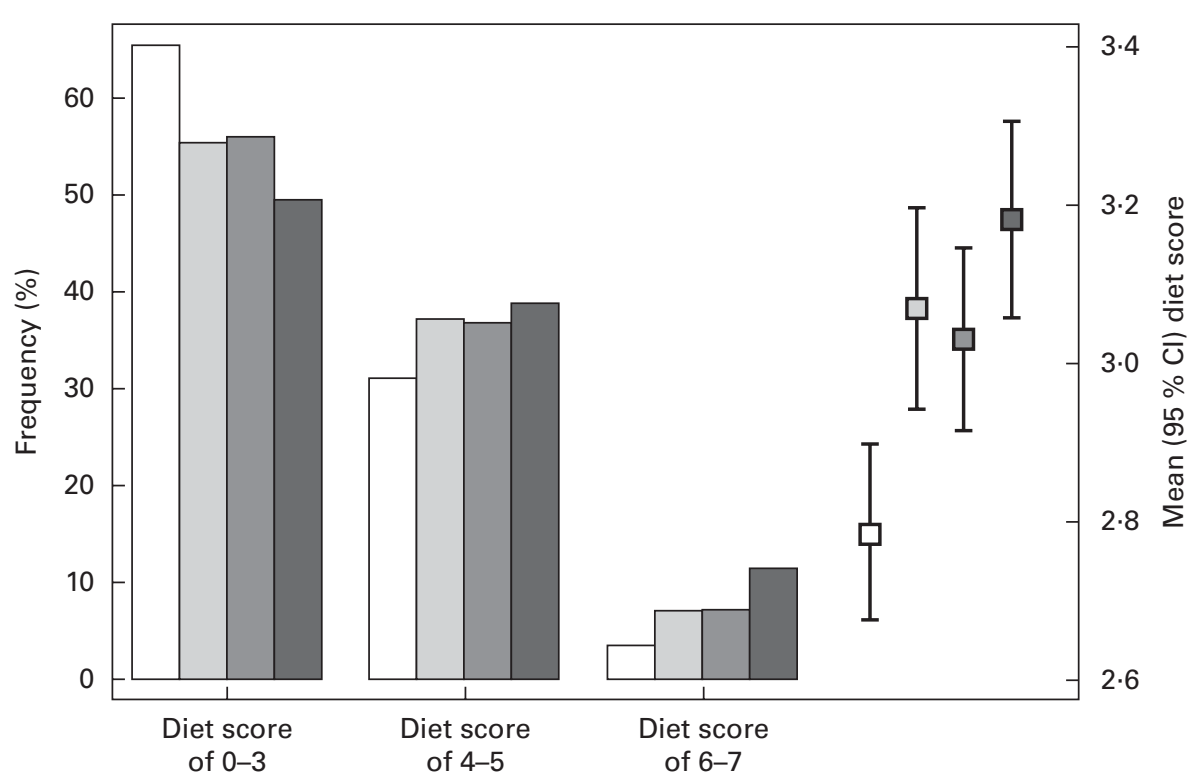

Fig. 2. Mediterranean diet (MD) score during pregnancy in the INMA (INfancia y Medio Ambiente) and RHEA mother-child cohort studies. ${ }^{*}$ The comparisons between mean MD scores across cohorts were performed using a one-way ANOVA test with Scheffe's correction for multiple comparisons: the only comparison not statistically significant was between the INMA-Sabadell and INMA-Valencia cohorts ( $P>0.05)$. $\square$, INMA-Gipuzkoa; $\square$, INMA-Sabadell; $\square$, INMA-Valencia; $\square$, RHEA.

estimate (RR $0 \cdot 83, \quad 95 \% \quad$ CI $\quad 0 \cdot 72, \quad 0 \cdot 97, \quad P$ for heterogeneity $=0.794)$. Further analysis by specific dairy products (milk, yogurt and cheese) revealed that high milk intake during pregnancy (third tertile $v$. first tertile of intake) was inversely associated with wheeze in the first year of life (meta-analysis pooled estimate: RR 0.82, 95\% CI 0.66, 1.00, $P$ for heterogeneity $=0.930$ ), while yogurt and cheese intakes were not associated with the risk of infantile wheeze and eczema. Maternal intake of fruits, vegetables, cereals and fats was not significantly associated with wheeze or eczema in the first year of life in the different cohorts and in the pooled estimate.

Because smoking during pregnancy has been previously associated with an increased risk of wheeze in infancy ${ }^{(31)}$, while smokers generally have poorer-quality diets than nonsmokers, with lower levels of antioxidant-rich foods such as fruits and vegetables ${ }^{(32)}$, we also looked for an interaction between smoking during pregnancy and adherence to a MD, but we found no significant interaction ( $P$ value for interaction $=0 \cdot 168$ ).

To evaluate the possibility of introducing confounding by total energy intake in pregnancy, physical activity during pregnancy, dampness exposure during infancy and pet ownership, we carried out additional analyses including these variables as possible confounders in the multivariate models, and the results remained essentially the same with the original analysis (data not shown).

\section{Discussion}

We evaluated MD adherence during pregnancy in two large population-based mother and child cohorts in Spain and Greece in association with wheeze and eczema in the first year of life. A high adherence to a MD during pregnancy was not significantly associated with infantile wheeze or eczema. A high intake of meat products during pregnancy was associated with an increased risk, while a high intake of dairy products was associated with a decreased risk of wheeze in the first year of life. To our knowledge, this is the first study to assess prospectively in general populations the impact of diet during pregnancy on wheeze and eczema in the first year of life.

The traditional MD refers to the dietary pattern in the Mediterranean olive grove areas at the beginning of the $1960 \mathrm{~s}^{\text {(33) }}$ This dietary pattern is low in SFA, rich in carbohydrates, fibre and antioxidants, and has a high content of MUFA and $n$-3 PUFA, which are primarily derived from olive oil and fish intake ${ }^{(34)}$. However, a MD presents regional variations derived from the same dietary pattern, influenced by various factors, such as socio-cultural, religious and economic determinants ${ }^{(35)}$. As expected, in the present study, the MD during pregnancy was not homogeneous, with women in the Atlantic area of Spain reporting higher intakes of fish products, while women in Crete reported higher intakes of fruits and nuts. This could explain in part the different results obtained in the present study across the different regions. For example, the heterogeneity of results on fish intake between the study areas could be explained by characteristics not included in the present analysis (such as subtypes of fish intake, persistent organic pollutants in fish products, other nutrients or even genetics).

Several recent reviews have stated the importance of a MD in the development of asthma in children ${ }^{(21,36,37)}$, while the ISAAC II multinational study reproduced these findings, showing that a MD in childhood protects against asthma symptoms ${ }^{(38)}$. Studies in adults have shown that a high adherence to a Mediterranean dietary pattern could also be associated with improved asthma control ${ }^{(39,40)}$. There are only three studies that have assessed the association between a MD during pregnancy and asthma outcomes in childhood 
(a)

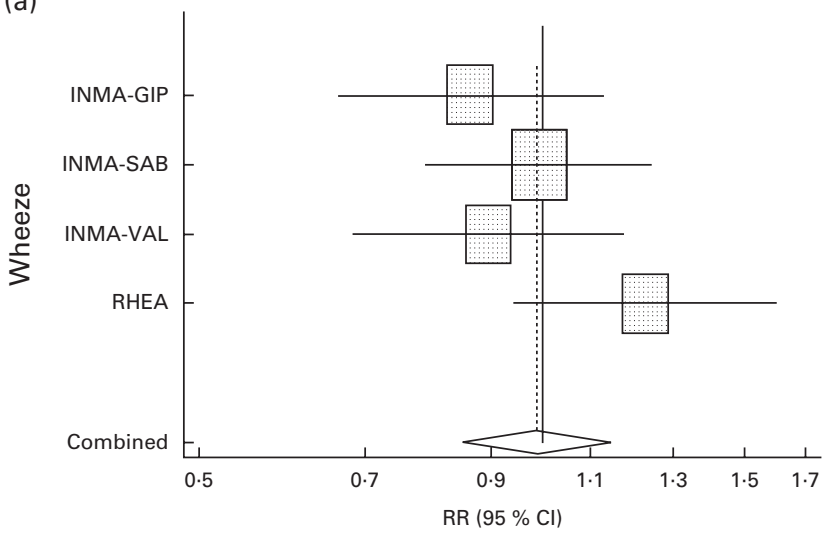

(c)

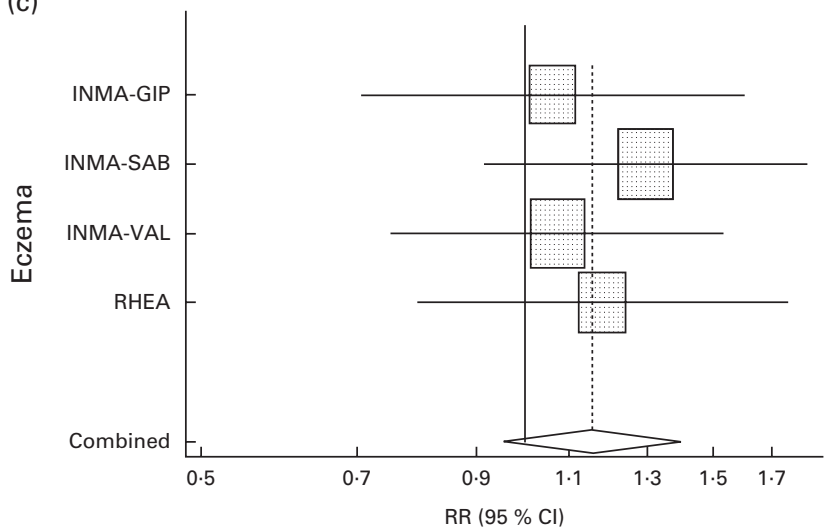

(b)

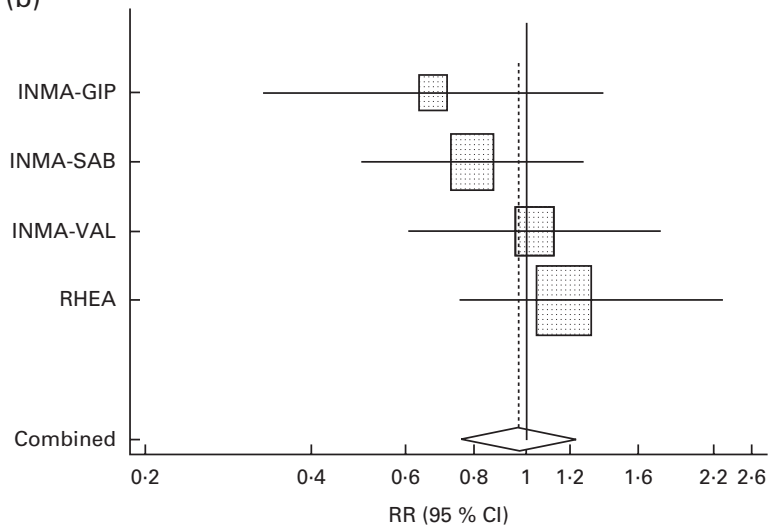

(d)

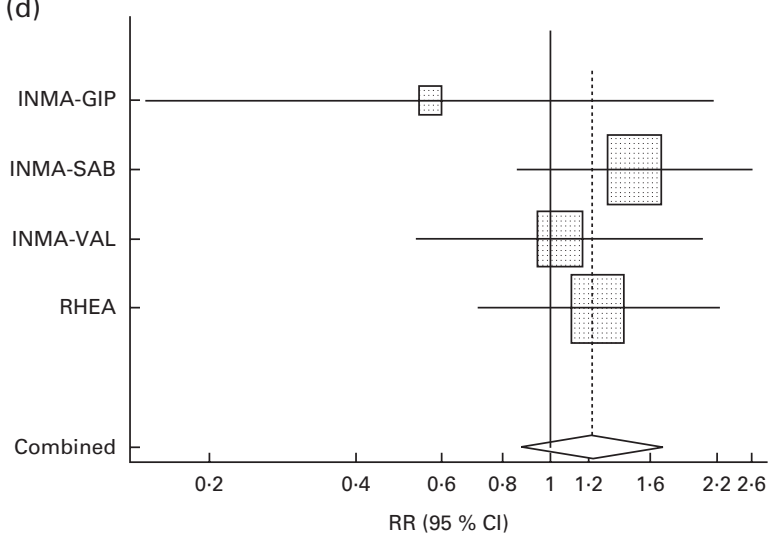

Fig. 3. Association between adherence to the Mediterranean diet (MD) in pregnancy and the risk of wheeze and eczema in the first year of life in the INMA (INfancia y Medio Ambiente) and RHEA mother-child cohort studies. Models were adjusted for maternal age, education, history of asthma, smoking during pregnancy, parity, duration of breast-feeding, child's age at assessment and child's sex. For eczema, maternal history of eczema is substituted for maternal history of asthma in the multivariate models. MD score: low (0-3); medium (4-5); high (6-8). Reference category: low MD score. Combined: summary estimates obtained by a random-effects meta-analysis. INMA-GIP, INMA-Gipuzkoa; INMA-SAB, INMA-Sabadell; INMA-VAL, INMA-Valencia. Medium MD score: (a) wheeze $I^{2}=25.8 \%, P=0.257$; (c) eczema $I^{2}=0.0 \%, P=0.858$. High MD score: (b) wheeze $I^{2}=0.0 \%, P=0.437 ;\left(\right.$ d) eczema $I^{2}=0.0 \%, P=0.581$. RR, relative risk.

with different results: Project Viva has shown no associations between MD adherence and asthma symptoms in children aged 3 years $^{(17)}$; the INMA-Menorca prospective motherchild cohort study has shown a protective effect of a high MD adherence during pregnancy on the risk of wheeze and atopy at 6.5 years ${ }^{(18)}$; de Batlle et al. ${ }^{(41)}$ have found no association between a MD during pregnancy and asthma or allergy outcomes in children at 6 years in Mexico, but in this study diet information was collected retrospectively 6 years after pregnancy. The different results obtained for these cohort studies may be explained by the fact that the categorisation of MD scores was based on the reported intake of foods; therefore, it is possible that a MD may translate to different food and nutrient intakes and account for different findings in cohorts that vary geographically. Moreover, the age of assessment was different in these studies, and this may have an impact on the estimated associations with diet.

The phenotype of wheeze in the first year of life is quite complex ${ }^{(42)}$. Many infants have episodes of wheeze associated with viral respiratory illnesses ${ }^{(43)}$. Neither the pathogenesis of these episodes nor their relationship with asthma has been completely elucidated ${ }^{(44)}$. Martinez et $a l^{(42)}$ suggested that most infants who wheeze in the first year of life have transient conditions associated with diminished airway function and have no increased risk of asthma or allergies later in life, while only in a minority of infants, early wheezing episodes are related to a predisposition to asthma. Thus, the results of the present study are more likely to reflect the effect of diet during pregnancy on transient wheezing in early life. Further follow-up of these cohorts will allow us to determine whether a higher adherence to a MD during pregnancy has an effect on childhood asthma in older ages.

A higher intake of meat and processed meat products during pregnancy was associated with an increased risk of wheeze in the first year of life. Meat is a primary source of SFA, while processed meat and meat products are rich dietary sources of industrially produced trans-fatty acids and several preservatives and colour fixatives, among which the most important are nitrites ${ }^{(45)}$. Industrial trans-fatty acids have been associated with increased inflammation and an increased risk of asthma and atopy ${ }^{(46)}$, while, on the other hand, ruminant trans-fatty acids in breast milk have been linked with a lower risk of eczema and allergic sensitisation in children before the age of 4 years ${ }^{(47,48)}$. Nitrites generate reactive nitrogen species that can amplify inflammatory processes in the airways and lung parenchyma, causing DNA damage, 
Table 2. Association between diet during pregnancy and the risk of wheeze and eczema in the first year of life in the INMA (INfancia y Medio Ambiente) and RHEA mother-child cohort studies*

(Relative risks (RR) and 95\% confidence intervals)

\begin{tabular}{|c|c|c|c|c|c|c|}
\hline & \multicolumn{3}{|c|}{ Wheeze } & \multicolumn{3}{|c|}{ Eczema } \\
\hline & $\mathrm{RR} \dagger$ & $95 \% \mathrm{Cl}$ & $P$ for heterogeneity & $\mathrm{RR} \dagger$ & $95 \% \mathrm{Cl}$ & $P$ for heterogeneity $\ddagger$ \\
\hline \multicolumn{7}{|l|}{ MD score§ } \\
\hline Medium & 0.99 & $0.85,1.15$ & 0.238 & $1 \cdot 16$ & $0.96,1.39$ & 0.858 \\
\hline High & 0.97 & $0.77,1.24$ & 0.407 & $1 \cdot 22$ & $0.88,1.70$ & 0.580 \\
\hline \multicolumn{7}{|c|}{ Food group intake } \\
\hline \multicolumn{7}{|c|}{ Fruits and nuts } \\
\hline $\mathrm{T} 2$ v. T1 & 0.95 & $0.81,1.12$ & 0.282 & 0.88 & $0.71,1.09$ & 0.813 \\
\hline T3 v. T1 & 0.94 & $0.81,1.09$ & 0.546 & 0.82 & $0.64,1.05$ & 0.304 \\
\hline \multicolumn{7}{|l|}{ Vegetables } \\
\hline T2 v. T1 & 0.87 & $0.76,1.01$ & 0.638 & $1 \cdot 18$ & $0.94,1.48$ & 0.874 \\
\hline T3 v. T1 & 0.94 & $0.79,1.12$ & 0.199 & $1 \cdot 21$ & $0.97,1.51$ & 0.763 \\
\hline \multicolumn{7}{|l|}{ Meat } \\
\hline T2 v. T1 & $1 \cdot 12$ & $0.92,1.35$ & 0.219 & $1 \cdot 13$ & $0.91,1.40$ & 0.811 \\
\hline T3 v. T1 & 1.22 & $1.00,1.49$ & 0.161 & 0.96 & $0.76,1.20$ & 0.990 \\
\hline \multicolumn{7}{|c|}{ Processed meat } \\
\hline $\mathrm{T} 2$ v. T1 & 1.03 & $0.89,1.20$ & 0.848 & 1.07 & $0.86,1.32$ & 0.814 \\
\hline T3 v. T1 & $1 \cdot 18$ & $1.02,1.37$ & 0.650 & 0.95 & $0.76,1.19$ & 0.445 \\
\hline \multicolumn{7}{|l|}{ Fish } \\
\hline T2 v. T1 & 0.95 & $0.82,1.11$ & 0.869 & 0.97 & $0.78,1.22$ & 0.735 \\
\hline T3 v. T1 & 1.08 & $0.84,1.38$ & 0.034 & 1.07 & $0.86,1.33$ & 0.979 \\
\hline \multicolumn{7}{|l|}{ Cereals } \\
\hline T2 v. T1 & 1.01 & $0.84,1.22$ & 0.189 & $1 \cdot 10$ & $0.89,1.37$ & 0.640 \\
\hline T3 v. T1 & 1.01 & $0.87,1.17$ & 0.812 & 1.07 & $0.82,1.41$ & 0.209 \\
\hline \multicolumn{7}{|c|}{ Dairy products } \\
\hline T2 v. T1 & 0.92 & $0.79,1.06$ & 0.439 & 1.01 & $0.81,1.26$ & 0.360 \\
\hline T3 v. T1 & 0.83 & $0.72,0.96$ & 0.794 & 0.95 & $0.76,1.18$ & 0.414 \\
\hline \multicolumn{7}{|l|}{ MUFA } \\
\hline T2 v. T1 & 1.09 & $0.94,1.26$ & 0.777 & $1 \cdot 30$ & $0.98,1.73$ & $0 \cdot 191$ \\
\hline T3 v. T1 & 1.06 & $0.79,1.40$ & 0.013 & 1.28 & $0.96,1.62$ & 0.512 \\
\hline \multicolumn{7}{|l|}{ SFA } \\
\hline T2 v. T1 & $1 \cdot 12$ & $0.95,1.32$ & 0.292 & 1.00 & $0.80,1.25$ & 0.546 \\
\hline T3 v. T1 & 1.08 & $0.93,1.26$ & 0.395 & 1.13 & $0.88,1.45$ & 0.284 \\
\hline
\end{tabular}

MD, Mediterranean diet; T1, tertile 1; T2, tertile 2; T3, tertile 3.

${ }^{*}$ Combined random effects derived from a meta-analysis.

† Models were adjusted for maternal age, education, history of asthma, smoking during pregnancy, parity, duration of breast-feeding, child's age at assessment and child's sex. For eczema, maternal history of eczema was used instead of maternal history of asthma in the multivariate models.

$\ddagger P$ value of the $\chi^{2}$ test of heterogeneity.

$\S M D$ score: low: 0-3; medium: 4-5; high: 6-8; reference category, low MD score.

inhibition of mitochondrial respiration and nitrosative stress ${ }^{(49)}$. The long-term persistence of nitrosative stress may contribute to the progressive deterioration of pulmonary function and may be implicated in the pathogenesis of wheeze and chronic obstructive pulmonary disease in adults ${ }^{(50,51)}$. Crosssectional studies have shown that a higher consumption of burgers in childhood was associated with an increased prevalence of asthma in the ISAAC study and two other cross-sectional studies ${ }^{(38,52,53)}$. The only birth cohort study that has assessed meat intake during pregnancy and the risk of wheeze and eczema in the first 2 years of life is that conducted in Japan among 763 mother-child pairs, and it reported a positive association between high maternal intake of meat during pregnancy and atopic eczema among infants aged 3-4 months ${ }^{(9,54)}$.

A high intake of dairy products, and specifically of milk, during pregnancy was significantly associated with a decreased risk of wheeze in the first year of life. For milk products, it is not clear whether associations should be attributed to the Ca content of the milk products or to the vitamin A content, or even may be due to microbial agents contained in the milk such as endotoxins or other factors characteristic of the farming environment ${ }^{(2,55)}$. Similar to the present findings, a birth cohort study in Japan has shown that a higher consumption of Ca and dairy foods during pregnancy is associated with a decreased risk of infantile wheeze (16-24 months) ${ }^{(56)}$, and the Prevention and Incidence of Asthma and Mite Allergy (PIAMA) birth cohort study in the Netherlands has shown an inverse association between maternal dairy product intake and childhood wheeze in the first 8 years of life, though not significant (OR 0.88, 95\% CI 0.71, 1.09) $)^{(57)}$. In the Danish National Birth Cohort, maternal whole milk and full-fat yogurt intakes during pregnancy were inversely associated with childhood asthma at 18 months, while low-fat yogurt intake was directly related to an increased risk of both childhood asthma and allergic rhinitis at this age ${ }^{(58)}$.

The strengths of the present study include the populationbased multi-centre prospective design, the large numbers of children with wheeze and eczema outcomes in the first year of life, the detailed data for dietary intake during pregnancy and the comprehensive assessment of potential confounding factors. The study population included women from the 
follow-up of several birth cohorts, giving us the opportunity to account for the effect of exposures assessed prospectively within the cohorts. As the INMA and RHEA studies used a different methodology for the estimation of food and nutrient intakes, we decided not to pool data from the four cohorts but to assess the impact of a MD in each cohort separately. We did not observe any substantial differences between the crude and the adjusted models. Thus, it is unlikely that over-adjustment affected our findings. The participants were unaware of the MD hypothesis being tested, so misclassification of intake estimated by the FFQ is likely to be random with respect to the outcomes.

There are several limitations in the present study that deserve acknowledgement. As in most studies on diet and health, we used self-reported diet during pregnancy and therefore information bias could have occurred. However, studies of nutrition in pregnancy have suggested that food frequency methods could present valid and reproducible estimates of dietary intakes in pregnant women ${ }^{(59)}$. Scores such as the MD score have certain limitations such as the variability in choosing cut-off points in the scores and the different distribution of the selected food groups in different populations ${ }^{(35)}$. A sensitivity analysis using a MD score in pregnancy as a continuous variable gave essentially the same results with the categorical analysis (data not shown). Although both the INMA and RHEA studies based their recorded information on the symptoms of wheeze and eczema in the ISAAC phase-I questionnaire, there are slight differences between the questions used in the two studies that mainly correspond to linguistic and cultural influences in the report of wheezing. We incorporated only breast-feeding as an indicator of postnatal diet in children, and it is possible that the children's diet may have an effect on the outcomes of interest. Unfortunately, we did not have available data on early-life exposure to drugs (i.e. paracetamol and antibiotics) that may increase the risk of eczema and wheeze in infancy ${ }^{(60-62)}$. A selection bias could be theoretically generated by the applied exclusion criteria and the loss to follow-up in the two cohorts. In the RHEA cohort, there were no significant differences regarding sociodemographic characteristics between the mother-child pairs who participated in the study and those who were excluded. The children lost to follow-up in the INMA cohort were from a lower social class, had a poorer paternal education and had a higher frequency of being preterm and having a low birth weight, but showed much less difference in terms of respiratory outcomes. This hampers the generalisation of the results to this population. However, we do not think that this would affect or bias the association between the exposure and respiratory outcomes of the children. Indeed, it might be helping us to better detect the effects of such exposure, because we avoided important factors related to respiratory impairment such as being a preterm child, having a low birth weight and coming from lower social and educational classes. Although we incorporated extensive information on potential social and environmental factors that are associated with wheeze and eczema in the first year of life, we acknowledge that there may be other factors linked with both outcomes and the MD during pregnancy that could explain this association.

In summary, these results suggest that adherence to a MD during pregnancy is not associated with infantile wheeze and eczema. High meat and processed meat intake during pregnancy could increase the risk of wheeze in the first year of life, while a high intake of dairy products may decrease it. The complex underlying processes that explain these findings require additional study. Further follow-up of these cohorts will allow us to determine whether a higher adherence to a MD during pregnancy has, in addition, an effect on childhood asthma.

\section{Acknowledgements}

The INMA study was funded by grants from Instituto de Salud Carlos III (Red INMA G03/176, CB06/02/0041, FIS-FEDER 03/ 1615, 04/1509, 04/1112, 04/1931, 05/1079, 05/1052, 06/1213, 07/0314 and 09/02647, FISS-PI042018, FISS-PI09/02311, FIS-PI06/0867, FIS-PS09/00090, FIS-PI041436, FIS-PI081151), the Conselleria de Sanitat Generalitat Valenciana, Generalitat de Catalunya-CIRIT 1999SGR 00241, Obra social Cajastur, Universidad de Oviedo, Department of Health of the Basque Government (2005111093 and 2009111069) and the Provincial Government of Gipuzkoa (DFG06/004 and DFG08/001). The RHEA study was partly supported by the European Union (EU) Integrated Projects (EU FP6-2003-Food-3-A NewGeneris, EU FP6; STREP Hiwate, EU FP7 ENV.2007.1.2.2.2; Project no. 211250 Escape, EU FP7-2008-ENV-1.2.1.4 Envirogenomarkers, EU FP7-HEALTH-2009-single-stage CHICOS, EU FP7 ENV.2008.1.2.1.6. Proposal no. 226285 ENRIECO) and the Greek Ministry of Health (Program of Prevention of obesity and neurodevelopmental disorders in preschool children, in Heraklion district, Crete, Greece: 2011-2014).

All authors made a substantial contribution to the study and they all personally reviewed and approved the submitted manuscript. Specifically, L. C. conceived the study, conducted the data analysis and prepared the manuscript; R. G. and T. R. carried out the statistical analysis and helped with the data interpretation and manuscript preparation; M. B., H. B. and C. I. supervised the data collection and helped with the manuscript preparation; J. V. designed the FFQ for the INMA cohort and contributed to the conceptual approach and manuscript preparation; M. K. provided feedback and critical revision of the manuscript and helped with the data interpretation and manuscript writing; J. S. supervised the data collection, provided critical review of the manuscript and helped with the data interpretation and manuscript preparation.

None of the authors declares a conflict of interest.

\section{References}

1. Allan K \& Devereux G (2011) Diet and asthma: nutrition implications from prevention to treatment. J Am Diet Assoc 111, 258-268.

2. Tricon S, Willers S, Smit HA, et al. (2006) Nutrition and allergic disease. Clin Experimen Aller Rev 6, 117-188. 
3. Varraso R (2012) Nutrition and asthma. Curr Allergy Asthma Rep 12, 201-210.

4. Willers SM, Devereux G, Craig LC, et al. (2007) Maternal food consumption during pregnancy and asthma, respiratory and atopic symptoms in 5-year-old children. Thorax $\mathbf{6 2}$, 773-779.

5. Miyake Y, Sasaki S, Tanaka K, et al. (2010) Consumption of vegetables, fruit, and antioxidants during pregnancy and wheeze and eczema in infants. Allergy 65, 758-765.

6. Romieu I, Torrent M, Garcia-Esteban R, et al. (2007) Maternal fish intake during pregnancy and atopy and asthma in infancy. Clin Exp Allergy 37, 518-525.

7. Sausenthaler S, Koletzko S, Schaaf B, et al. (2007) Maternal diet during pregnancy in relation to eczema and allergic sensitization in the offspring at $2 \mathrm{y}$ of age. Am J Clin Nutr 85, $530-537$.

8. Salam MT, Li YF, Langholz B, et al. (2005) Maternal fish consumption during pregnancy and risk of early childhood asthma. J Asthma 42, 513-518.

9. Miyake Y, Sasaki S, Tanaka K, et al. (2009) Maternal fat consumption during pregnancy and risk of wheeze and eczema in Japanese infants aged 16-24 months: the Osaka Maternal and Child Health Study. Thorax 64, 815-821.

10. Martindale S, McNeill G, Devereux G, et al. (2005) Antioxidant intake in pregnancy in relation to wheeze and eczema in the first two years of life. Am J Respir Crit Care Med 171, 121-128.

11. Litonjua AA, Rifas-Shiman SL, Ly NP, et al. (2006) Maternal antioxidant intake in pregnancy and wheezing illnesses in children at $2 \mathrm{y}$ of age. Am J Clin Nutr 84, 903-911.

12. Devereux G, Turner SW, Craig LC, et al. (2006) Low maternal vitamin $\mathrm{E}$ intake during pregnancy is associated with asthma in 5-year-old children. Am J Respir Crit Care Med 174, 499-507.

13. Camargo CA Jr, Rifas-Shiman SL, Litonjua AA, et al. (2007) Maternal intake of vitamin D during pregnancy and risk of recurrent wheeze in children at $3 \mathrm{y}$ of age. Am J Clin Nutr 85, 788-795.

14. Devereux G, Litonjua AA, Turner SW, et al. (2007) Maternal vitamin $D$ intake during pregnancy and early childhood wheezing. Am J Clin Nutr 85, 853-859.

15. Jacobs DR Jr \& Steffen LM (2003) Nutrients, foods, and dietary patterns as exposures in research: a framework for food synergy. Am J Clin Nutr 78, 508S-513S.

16. Shaheen SO, Northstone K, Newson RB, et al. (2009) Dietary patterns in pregnancy and respiratory and atopic outcomes in childhood. Thorax 64, 411-417.

17. Lange NE, Rifas-Shiman SL, Camargo CA, et al. (2010) Maternal dietary pattern during pregnancy is not associated with recurrent wheeze in children. J Allergy Clin Immunol 126, 250-255, 255.e1-255.e4.

18. Chatzi L, Torrent M, Romieu I, et al. (2008) Mediterranean diet in pregnancy is protective for wheeze and atopy in childhood. Thorax 63, 507-513.

19. Miyake Y, Okubo H, Sasaki S, et al. (2011) Maternal dietary patterns during pregnancy and risk of wheeze and eczema in Japanese infants aged 16-24 months: the Osaka Maternal and Child Health Study. Pediatr Allergy Immunol 22, 734-741.

20. Guxens M, Ballester F, Espada M, et al. (2012) Cohort Profile: The INMA - INfancia y Medio Ambiente - (Environment and Childhood) Project. Int J Epidemiol 41, 930-940.

21. Chatzi L \& Kogevinas M (2009) Prenatal and childhood Mediterranean diet and the development of asthma and allergies in children. Public Health Nutr 12, 1629-1634.
22. Regidor E, Martinez D, Calle ME, et al. (2008) Socioeconomic patterns in the use of public and private health services and equity in health care. BMC Health Serv Res $\mathbf{8}, 183$.

23. Davey GK, Spencer EA, Appleby PN, et al. (2003) EPIC-Oxford: lifestyle characteristics and nutrient intakes in a cohort of 33883 meat-eaters and 31546 non meat-eaters in the UK. Public Health Nutr 6, 259-269.

24. Chatzi L, Plana E, Daraki V, et al. (2009) Metabolic syndrome in early pregnancy and risk of preterm birth. Am J Epidemiol 170, 829-836.

25. Willett WC, Sampson L, Stampfer MJ, et al. (1985) Reproducibility and validity of a semiquantitative food frequency questionnaire. Am J Epidemiol 122, 51-65.

26. Vioque J, Navarette-Muñoz EM, Gimerez-Monzó D, et al. (2013) Reproducibility and validity of a food frequency questionnaire among pregnant women in a Mediterranear area. Nutr J 12, 26.

27. Chatzi L, Melaki V, Sarri K, et al. (2011) Dietary patterns during pregnancy and the risk of postpartum depression: the mother-child 'Rhea' cohort in Crete, Greece. Public Health Nutr 14, 1663-1670.

28. Trichopoulou A, Costacou T, Bamia C, et al. (2003) Adherence to a Mediterranean diet and survival in a Greek population. $N$ Engl J Med 348, 2599-2608.

29. Chatzi L, Mendez M, Garcia R, et al. (2012) Mediterranean diet adherence during pregnancy and fetal growth: INMA (Spain) and RHEA (Greece) mother-child cohort studies. Br J Nutr 107, 135-145.

30. Anonymous (1998) Worldwide variations in the prevalence of asthma symptoms: the International Study of Asthma and Allergies in Childhood (ISAAC). Eur Respir $J$ 12, $315-335$.

31. Pattenden S, Antova T, Neuberger M, et al. (2006) Parental smoking and children's respiratory health: independent effects of prenatal and postnatal exposure. Tob Control 15, 294-301.

32. Palaniappan U, Jacobs Starkey L, O'Loughlin J, et al. (2001) Fruit and vegetable consumption is lower and saturated fat intake is higher among Canadians reporting smoking. J Nutr 131, 1952-1958.

33. Willett WC, Sacks F, Trichopoulou A, et al. (1995) Mediterranean diet pyramid: a cultural model for healthy eating. Am J Clin Nutr 61, 1402S-1406S.

34. Trichopoulou A \& Lagiou P (1997) Healthy traditional Mediterranean diet: an expression of culture, history, and lifestyle. Nutr Rev 55, 383-389.

35. Bach A, Serra-Majem L, Carrasco JL, et al. (2006) The use of indexes evaluating the adherence to the Mediterranean diet in epidemiological studies: a review. Public Health Nutr 9, $132-146$.

36. Bloomberg GR (2011) The influence of environment, as represented by diet and air pollution, upon incidence and prevalence of wheezing illnesses in young children. Curr Opin Allergy Clin Immunol 11, 144-149.

37. Nurmatov U, Devereux G \& Sheikh A (2011) Nutrients and foods for the primary prevention of asthma and allergy: systematic review and meta-analysis. J Allergy Clin Immunol 127, 724-733.e1-e30.

38. Nagel G, Weinmayr G, Kleiner A, et al. (2010) Effect of diet on asthma and allergic sensitisation in the International Study on Allergies and Asthma in Childhood (ISAAC) Phase Two. Thorax 65, 516-522.

39. Barros R, Moreira A, Fonseca J, et al. (2008) Adherence to the Mediterranean diet and fresh fruit intake are associated with improved asthma control. Allergy 63, 917-923. 
40. Barros R, Moreira A, Fonseca J, et al. (2011) Dietary intake of alpha-linolenic acid and low ratio of $n-6: n-3$ PUFA are associated with decreased exhaled $\mathrm{NO}$ and improved asthma control. Br J Nutr 106, 441-450.

41. de Batlle J, Garcia-Aymerich J, Barraza-Villarreal A, et al. (2008) Mediterranean diet is associated with reduced asthma and rhinitis in Mexican children. Allergy 63, 1310-1316.

42. Martinez FD, Wright AL, Taussig LM, et al. (1995) Asthma and wheezing in the first six years of life. The Group Health Medical Associates. N Engl J Med 332, 133-138.

43. Wright AL, Taussig LM, Ray CG, et al. (1989) The Tucson Children's Respiratory Study. II. Lower respiratory tract illness in the first year of life. Am J Epidemiol 129, 1232-1246.

44. Samet JM, Tager IB \& Speizer FE (1983) The relationship between respiratory illness in childhood and chronic airflow obstruction in adulthood. Am Rev Respir Dis 127, $508-523$

45. Jakszyn P, Agudo A, Ibanez R, et al. (2004) Development of a food database of nitrosamines, heterocyclic amines, and polycyclic aromatic hydrocarbons. J Nutr 134, 2011-2014.

46. Weiland SK, von Mutius E, Husing A, et al. (1999) Intake of trans fatty acids and prevalence of childhood asthma and allergies in Europe. ISAAC Steering Committee. Lancet 353, 2040-2041.

47. Thijs C, Muller A, Rist L, et al. (2011) Fatty acids in breast milk and development of atopic eczema and allergic sensitisation in infancy. Allergy 66, 58-67.

48. Wijga AH, van Houwelingen AC, Kerkhof M, et al. (2006) Breast milk fatty acids and allergic disease in preschool children: the Prevention and Incidence of Asthma and Mite Allergy birth cohort study. J Allergy Clin Immunol 117, 440-447.

49. Ricciardolo FL, Di Stefano A, Sabatini F, et al. (2006) Reactive nitrogen species in the respiratory tract. Eur J Pharmacol $\mathbf{5 3 3}, 240-252$

50. Kharitonov SA \& Barnes PJ (2003) Nitric oxide, nitrotyrosine, and nitric oxide modulators in asthma and chronic obstructive pulmonary disease. Curr Allergy Asthma Rep 3, 121-129.

51. Varraso R, Jiang R, Barr RG, et al. (2007) Prospective study of cured meats consumption and risk of chronic obstructive pulmonary disease in men. Am J Epidemiol 166, 1438-1445.
52. Wickens K, Barry D, Friezema A, et al. (2005) Fast foods are they a risk factor for asthma? Allergy 60, 1537-1541.

53. Garcia-Marcos L, Canflanca IM, Garrido JB, et al. (2007) Relationship of asthma and rhinoconjunctivitis with obesity, exercise and Mediterranean diet in Spanish schoolchildren. Thorax 62, 503-508.

54. Saito K, Yokoyama T, Miyake Y, et al. (2010) Maternal meat and fat consumption during pregnancy and suspected atopic eczema in Japanese infants aged 3-4 months: the Osaka Maternal and Child Health Study. Pediatr Allergy Immunol 21, 38-46.

55. Riedler J, Braun-Fahrlander C, Eder W, et al. (2001) Exposure to farming in early life and development of asthma and allergy: a cross-sectional survey. Lancet 358, 1129-1133.

56. Miyake Y, Sasaki S, Tanaka K, et al. (2010) Dairy food, calcium and vitamin D intake in pregnancy, and wheeze and eczema in infants. Eur Respir J 35, 1228-1234.

57. Willers SM, Wijga AH, Brunekreef B, et al. (2008) Maternal food consumption during pregnancy and the longitudinal development of childhood asthma. Am J Respir Crit Care Med 178, 124-131.

58. Maslova E, Halldorsson TI, Strom M, et al. (2012) Low-fat yoghurt intake in pregnancy associated with increased child asthma and allergic rhinitis risk: a prospective cohort study. J Nutr Sci (Epublication ahead of print version 6 July 2012).

59. Meltzer HM, Brantsaeter AL, Ydersbond TA, et al. (2008) Methodological challenges when monitoring the diet of pregnant women in a large study: experiences from the Norwegian Mother and Child Cohort Study (MoBa). Matern Child Nutr 4, 14-27.

60. Goksor E, Thengilsdottir H, Alm B, et al. (2011) Prenatal paracetamol exposure and risk of wheeze at preschool age. Acta Paediatr 100, 1567-1571.

61. Kummeling I, Stelma FF, Dagnelie PC, et al. (2007) Early life exposure to antibiotics and the subsequent development of eczema, wheeze, and allergic sensitization in the first 2 years of life: the KOALA Birth Cohort Study. Pediatrics 119, e225-e231.

62. Wennergren $G$ (2011) Paracetamol - accumulating reports of an association with allergy and asthma. Acta Paediatr 100, $12-13$. 